\title{
The different faces of pulmonary hydatid disease
}

\author{
S A Thula, FCPaeds; M Ndlovu, MB BCh; R Masekela, PhD \\ Division of Paediatric Pulmonology, Department of Maternal and Child Health, Nelson R Mandela School of Clinical Medicine, University of KwaZulu-Natal, \\ Durban, South Africa
}

Corresponding author: R Masekela (masekelar@ukzn.ac.za)

\begin{abstract}
Hydatid disease is responsible for causing cystic disease. In children, it classically involves the liver, lung and brain but can involve almost any organ or numerous organs simultaneously. The lung is the most common target organ in children, while in adults hepatic involvement is more common. We present three case reports of children who presented with hydatid disease with varying clinical manifestations of pulmonary hydatidosis and complications, and their related management.
\end{abstract}

S Afr Respir J 2016;22(2):43-45. DOI:10.7196/SARJ.2016.v22i2.74

\section{Case presentations}

Case 1

The first case is an 11-year-old boy referred to our hospital for the assessment of ring lesions seen on X-ray. He was from a rural area of KwaZulu-Natal Province. He had a 3-month history of cough, fever and loss of appetite. He had no history of tuberculosis (TB) contact. On examination he was wasted, had tachypnoea and had bilateral crackles in both lung fields. The rest of the system examination was normal. His chest $\mathrm{X}$-rays showed bilateral well-circumscribed round lesions (Fig. 1). The enzyme-linked immunosorbent assay (ELISA) HIV test was negative and sputum microscopy sensitivity and culture revealed no pathogens. The hydatid indirect haemagglutinin assay was positive and the full blood count smear showed eosinophilia. A diagnosis of bilateral diffuse echinococcosis was made. The thoracic surgeons were consulted; however, because of the extent of the disease, with multiple lesions bilaterally, the agreed therapeutic option was a trial of medical treatment, as the lesions were inoperable. Medical management was then instituted with albendazole $400 \mathrm{mg}$ /day for 6 months. The follow-up X-rays showed dramatic improvement of the lesions and good resolution.

\section{Case 2}

A 5-year-old boy was referred from rural Eastern Cape Province for assessment of a suspected non-resolving pleural effusion. The parents gave a history of progressively worsening symptoms of a tight chest and breathlessness on exertion over the past 3

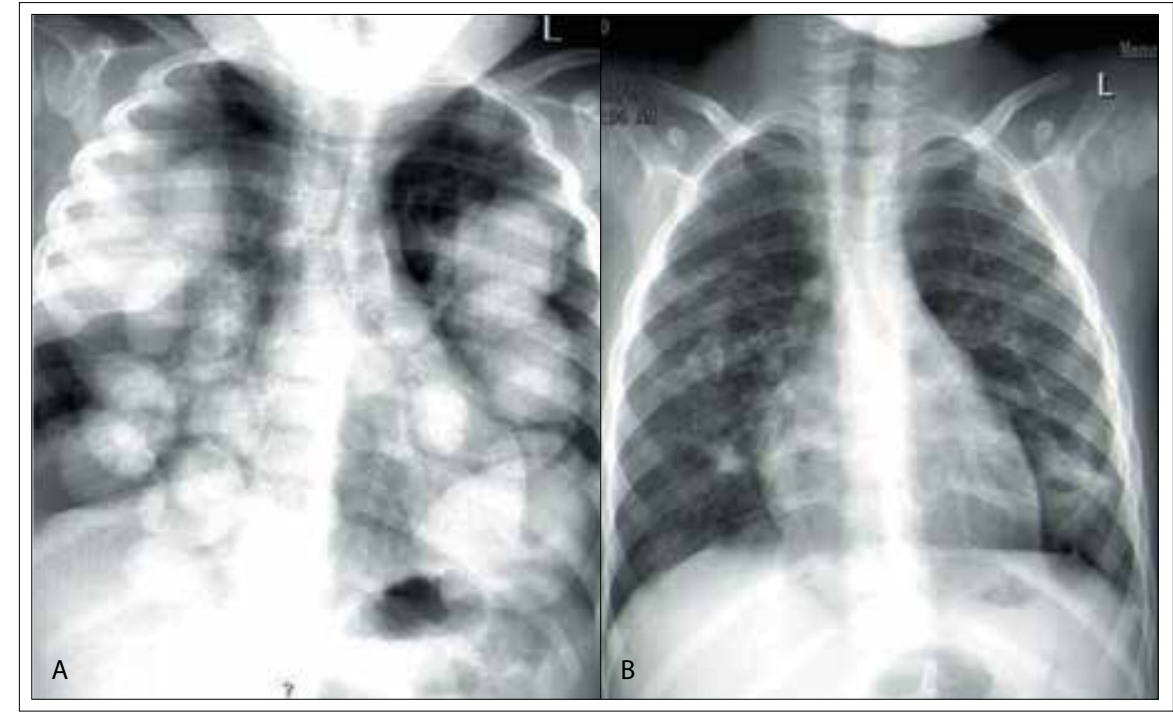

Fig. 1. Chest X-rays of Case 1: (A) pre-treatment; and (B) post-treatment.

years. He had been diagnosed with asthma by numerous general practitioners but the symptoms had not improved on treatment. On clinical examination, he had respiratory distress with tachypnoea, bilateral wheezes over both lung fields and decreased air entry. The remaining systems were normal. The chest X-ray revealed an almost complete white-out of the right hemithorax with an air-fluid level (Fig. 2). He had been suspected of having a pleural effusion/empyema at the referral hospital and this had been drained, with clear blood-tinged fluid aspirated. The full blood count smear showed eosinophilia. The hydatid indirect haemagglutination assay was highly positive. Computed tomography (CT) scan confirmed the presence of a large cystic lesion with a thick wall and air-fluid levels (Fig. 3). Ultrasound did not show any

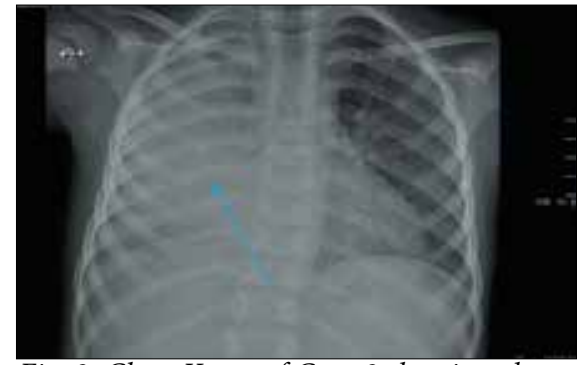

Fig. 2. Chest $X$-ray of Case 2 showing almost complete white-out of the right hemithorax and an air-fluid level, as well as obliteration of the right main bronchus. The blue arrow indicates a large cyst involving the entire right hemithorax with no midline shift.

lesions in the liver. The child was referred to cardiothoracic surgeons, the lesion operated and a cyst removed (Fig. 4). 


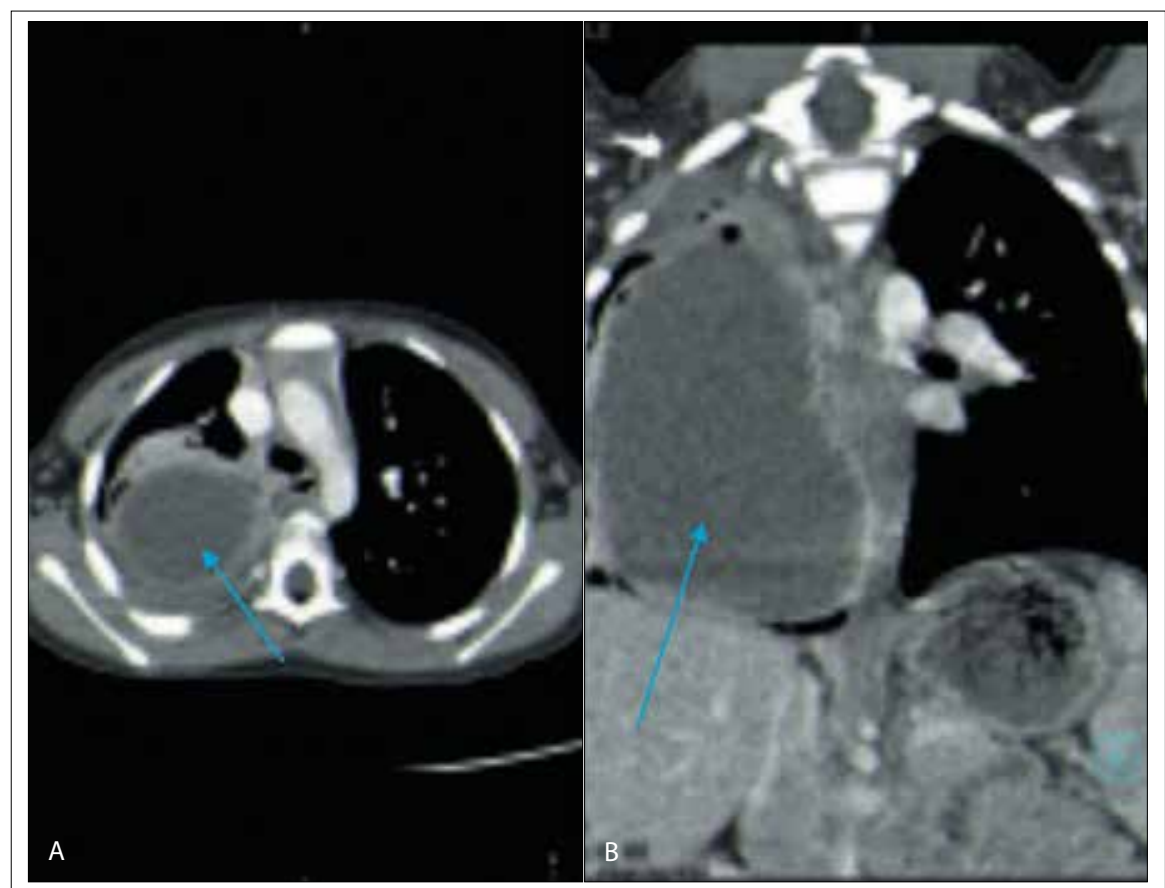

Fig. 3. Axial (A) and coronal (B) views showing large, well-circumscribed cystic lesion in the right hemithorax with air-fluid levels. (A) CT axial view with arrow demonstrating thick-walled rightsided cyst. (B) Coronal view with arrow demonstrating large right-lung cyst.

\section{Case 3}

The final case was a 12-year-old male from rural Eastern Cape, referred for the assessment of a mediastinal mass and a query of hydatid cyst. The history was that of weight loss and chest pain present for 3 months. There was no history of TB contact and the child was confirmed as HIV-negative. On clinical examination he had a respiratory rate of 25 breaths per minute with a symmetric chest shape, poor air entry bilaterally and dullness to percussion. Chest X-ray showed bilateral masses, with a large circular lesion occupying almost the entire right hemithorax and a smaller round lesion in the left upper lobe with air-fluid levels (Fig. 5). CT scan confirmed multiple cystic masses in the left and right hemithoraces. Full blood count revealed eosinophilia and the Echinococcus indirect haemagglutination assay was positive. Thoracic surgeons were consulted and during surgery it was evident that the left-side cyst was infected and collapsed (Fig. 6). The patient received albendazole for 1 month post surgery. The left-side cyst was removed a month later.

\section{Discussion}

Pulmonary hydatid disease is caused by the tapeworm Echinococcus. The two common types of Echinococcus prevalent in South Africa (SA) are E. granulosus and E. multilocularis. The life cycle of these worms includes a definitive and intermediate host (Table 1) and Echinococcus infection is found worldwide. In some parts of Canada (Ontario) about 50\% of moose are infected with the parasite, while dog infestation is estimated to be $28-50 \%$ in the same area. ${ }^{[1]}$ In SA, the disease is prevalent in Limpopo, Eastern Cape, North West and Northern Cape provinces. ${ }^{[2]}$ Retrospective National Health Laboratory Service information system results for serology, microscopy and histopathology from eight provinces (excluding KwaZulu-Natal) gave an overall positivity rate in submitted diagnostic samples of $17.0 \%$ (1 056/6 211), with Eastern Cape leading at $30.4 \%$, North West $19.0 \%$ and Northern Cape $18.0 \%{ }^{[3,4]}$

The life cycle of the Echinococcus worm begins with the host animal shedding, via its faeces, a mature segment full of eggs. The eggs are picked up by herbivores (intermediate host), where the eggs hatch in the small intestine and grow to a larval stage, known as an oncosphere. Oncospheres penetrate the small intestine and are then transported to the liver and other organs via the portal and lymphatic systems. The oncospheres grow into cysts in the organs of the intermediate host. The cysts enlarge

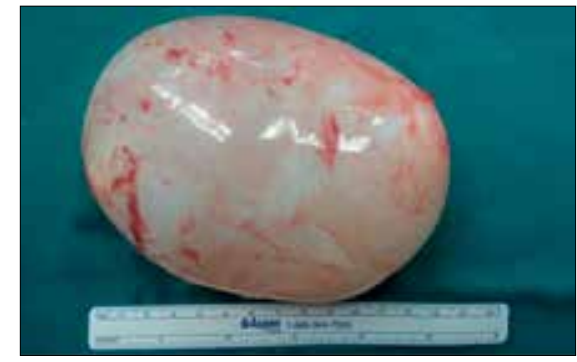

Fig. 4. Cyst removed surgically in Case 2, measuring $13 \mathrm{~cm}$.

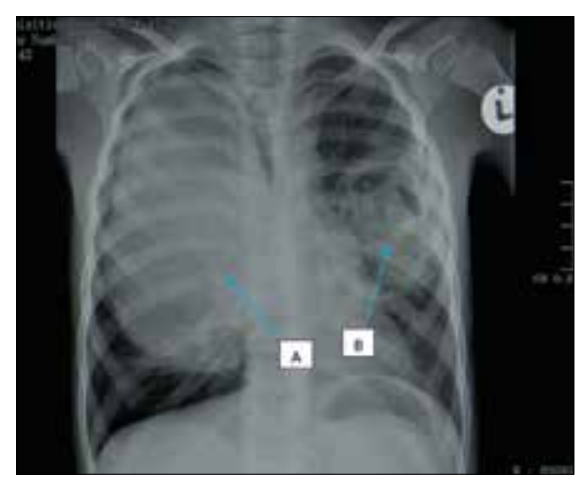

Fig. 5. Chest X-ray of Case 3: (A) arrow shows a large, intact, right-sided, well-circumscribed cyst; and (B) arrow shows a ruptured left-sided cyst with air-fluid levels.

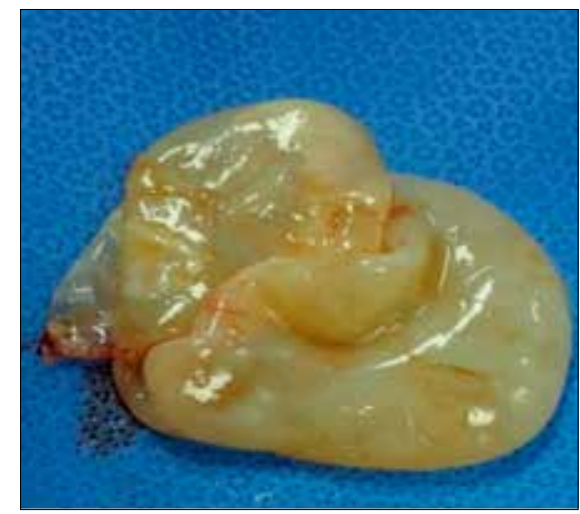

Fig. 6. Collapsed right-sided cyst on removal.

slowly and create protoscolices and daughter cysts. The definitive hosts are infected by eating the infected organs of the intermediate host. The protoscolices grow into adult worms on reaching the small intestine of the host animal. Handling soil or consuming drinking water infested by the eggs may incidentally infect humans. The larvae penetrate the intestines, and enter the portal and lymphatic systems into the liver and other organs.

Pulmonary echinococcosis is the most common manifestation and presentation site for children with hydatid disease and may present as either alveolar or mediastinal 
Table 1. The life cycle of two different types of Echinococcus worm and hosts involved

\begin{tabular}{lll}
\hline Organism & Definitive host & Intermediate host \\
\hline E. granulosus & Dogs and other canines & Sheep and goats, other herbivores \\
E. multilocularis & Dogs and foxes & Rodents
\end{tabular}

disease, or both. These cases illustrate the difficulty that may confront a doctor in approaching the patient with hydatid disease. The presentation may mimic other common diseases such as TB and asthma, and the doctor should be aware of these, with a high index of suspicion in patients who present with cystic lesions. The vast majority of pulmonary cysts in children are congenital. In endemic areas, Echinococcus must always be in the differential diagnosis as a secondary cause of lung or mediastinal cysts. ${ }^{[4]}$

The diagnosis of Echinococcus infection is usually made by serological tests. Indirect haemagglutination test and indirect fluorescent antibody tests have sensitivity ranging from $60 \%$ to $90 \%$; therefore, patients may be missed using these tests. ${ }^{[6]}$ ELISA assays were found to have a high sensitivity at $97 \%$ but are poor in differentiating the serological infection type. ${ }^{[5]}$ Radiological investigations should include a chest X-ray, and chest CT scan where complications are suspected, in unusual presentations or for presurgical planning. Liver cysts can be diagnosed on ultrasonography.

Chest X-ray features include solitary or multiple cysts in the lung or in the middle mediastinum. CT scan features include multiple or solitary cysts with well-defined borders, in uncomplicated cases. The contents are hypodense when compared with the capsule.

In complicated cases, CT scan may present with the following features: ${ }^{[6]}$

- Meniscus or air crescent sign: describes the crescent of air that can be seen in the hydatid cyst. This usually results during enlargement of the cyst, when the cyst ruptures into an adjacent bronchus and air enters between the pericyst and endocyst.

- Cumbo or onion-peel sign: air between the endocyst and pericyst in addition to rupture of the cyst with air tracking into the endocyst. This creates a double air layer appearance.

- Water-lily sign: detachment of the endocyst membrane resulting in floating membranes within the pericyst that mimic the appearance of a water lily.

The treatment of choice is surgical enucleation of the cyst. Where this is not possible, as in our first case report, medical therapy with albendazole should be considered as first-line treatment. Chemoprophylaxis with albendazole is also indicated pre- and postsurgical intervention. Another important learning point is that in patients treated for suspected pleural effusions, as in the second case report, it is critical that an alternative diagnosis is entertained on non-resolution of symptoms, and further investigations should be performed.

The prognosis of hydatid disease depends on a number of factors, and is better with low internal complexity of the cyst and small cyst size. With proper diagnosis and treatment, outcomes are good.

\section{Conclusion}

Healthcare providers working in areas with high prevalence of hydatidosis should be aware of the possibility of this condition being present in patients presenting with respiratory complaints. Other target organ involvement should be excluded, particularly in adults.

\section{References}

1. Grosso G, Gruttadaria S, Biondi A, Marventano S, Mistretta A. Worldwide epidemiology of liver hydatidosis including the Mediterranean area. World J Gastroenterol 2012;18(13):1425-1437. DOI:10.3748/wjg.v18.i13.1425

2. Wahlers K, Menezes CN, Wong ML, Zeyhle E, Ahmed $\mathrm{ME}$, et al. Cystic echinococcus in sub-Saharan Africa. Lancet Infect Dis 2012;12(11);871-880. DOI:10.1016/ s1473-3099(12)70155-x

3. Mogoye KB. Human Echinococcus in South Africa. MMed dissertation, Johannesburg: University of the Witwatersrand, 2013.

4. Mogoye B, Menezes CN, Grobusch MP, Wahlers K, Frean J. Human cystic echinococcosis in South Africa. Onderstepoort J Vet Res 2012;79(2):e1. DOI:10.4102/ ojvr.v79i2.469

5. Centers for Disease Control. Echinococcus. http:// www.cdc.gov/parasites/echinococcus/ (accessed 30 March 2016).

6. Morar R, Feldman C. Pulmonary echinococcus. Eur Resp J 2003;21:1069-1077. DOI:10.1183/09031936.03 .00108403 